\title{
PACAP-induced pain responses are mediated by spinal Cyr61-chemokine signaling pathway
}

\section{Tomoki Ogata ${ }^{1}$, Tatsunori Maeda ${ }^{1}$, Masafumi Yokai $^{2}$, Atsuro Miyata ${ }^{2}$, Takashi Kurihara ${ }^{2}$, Ichiro Takasaki ${ }^{1}$}

${ }^{I}$ Grad. Sch. Sci. \& Eng. Res., univ. Toyama, Japan, ${ }^{2}$ Grad. Sch. Med. \& Dent. Sci., Kagoshima Univ., Japan

Neuropathic pain such as post-herpetic neuralgia and trigeminal neuralgia is a chronic pain characterized by severe spontaneous pain and allodynia, however, the mechanisms of the pain are unclear. Recently, we found that intrathecal (i. t.) injection of pituitrary adenylate cyclase-activating polypeptide (PACAP) or maxadilan (a selective PAC1 receptor agonist) induces long-term mechanical allodynia in mice and that such treatment increase the expression level of cysteine rich protein 61 (Cyr61) mRNA in the spinal cord. Cyr61 is a multifunctional matricellular protein that plays important roles in development, inflammation, tissue repair, fibrosis, and cancer. However, the involvement of Cyr61 on pain has not been reported. Therefore, in order to clarify the involvement of spinal Cyr61 in PACAP-induced mechanical allodynia, in this study, we investigated 1) the expression of Cyr61 in the spinal cord, 2) pain-like responses induced by i.t. Cyr61, and 3) the involvement of chemokines and its receptors in Cyr61-induced pain-like responses.

We found that i.t. injection of PACAP markedly increased the Cyr61-like immunoreactivities in the NeuN-positive neurons, but not in the GFAP-positive astrocytes in the dorsal horn of the spinal cord. I.t. injection of recombinant Cyr61 (rCyr61, $2.4 \mathrm{pmol}$ ) induced mechanical allodynia in mice which was lasted at least for 2 weeks. Next we examined the expression of CC chemokine mRNA in the spinal cord after i.t. injection of rCyr61. As a results, CCL2, CCL7, and CCL22 mRNA expression was significantly elevated after i.t. injection of $\mathrm{rCyr61}$. rCyr61 also significantly increased the expression of CCL2, CCL7, and CCL22 mRNA in cultured primary astrocyte and KT-5 cells (the mouse astrocyte cell line). Finally, we examined the effects of antagonist of chemokine receptors on rCyr61-induced mechanical allodynia. Although the antagonists of CCR1 (J113863), CCR3 (SB328437) or CCR5 (Maraviroc) had no effects, we found that i.t. injection of CCR2 antagonist (RS504393, $1 \mu \mathrm{mol}$ ) inhibited rCyr61- induced mechanical allodynia.

The present results suggest that neuron-derived Cyr61-CC chemokine-CCR2 signaling pathways in the spinal cord are deeply involved in the PACAP-induced pain responses. 